\title{
Temps et idéologie : Marlowe, Shakespeare
}

\section{Gisèle Venet}

Marie-Thérèse Jones-Davies (éd.)

\section{(2) OpenEdition}

\section{Journals}

Édition électronique

URL : http://journals.openedition.org/shakespeare/433

DOI : $10.4000 /$ shakespeare.433

ISSN : 2271-6424

Éditeur

Société Française Shakespeare

Édition imprimée

Date de publication : 1 novembre 1981

Pagination : 69-78

ISBN : 2-86433-016-4

Référence électronique

Gisèle Venet, "Temps et idéologie : Marlowe, Shakespeare », Actes des congrès de la Société française Shakespeare [En ligne], 3 | 1981, mis en ligne le 01 novembre 2007, consulté le 20 janvier 2020. URL: http://journals.openedition.org/shakespeare/433 ; DOI : 10.4000/shakespeare.433 


\title{
THEATRE ET IDEOLOGIES :
} Marlowe, Shakespeare

\author{
DIRECTEUR DE LA PUBLICATION \\ M.T. Jones - Davies
}

JEAN TOUZOT Libraire - Editeur

38 , rue Saint-Sulpice 75278 PARIS CEDEX 061982 
1982 Jean Touzot Libiaire-Editeur, Paris.

Reproduction et traduction mzme partielle interdite.

Tous droits réservts potir lous pays

y compris i'U,R.S.S. et les pays scandinaves

ISBN 2-86433-016-4 


\title{
AVANT-PROPOS
}

L'opposition soulignée par Horace entre éthique et esthétique Aut prodesse volunt aut delectare poetae - que les Elisabéthains aiment à évoquer, pourrait être le lien qui rattache les unes aux autres les communications, rencontres et table ronde de notre troisième congrès (1981) sur le thème : "Théátre et Idéologies: Marlowe, Shakespeare.» Toutefois, le résultat de nos échanges montre un dépassement possible de l'alternative éthique / esthétique, puisqu'il met en valeur l'insertion d'idéologies susceptibles de transformer la fonction même de la littérature.

Les pièces analysées dans le contexte de leur temps révèlent de multiples aspects de la vision du monde et de la place que l'homme $y$ occupe, tels que les projette le théâtre anglais au tournant du XVIe et au début du XVIIe siècle.

Une exploration du langage et de la pensée des deux grands dramaturges aboutit à un commentaire sur les courants philosophiques ou religieux, sur les idées parfois contradictoires et sur la curiosité intellectuelle jamais assouvie qui se font jour à travers la rhétorique de la scène et le jeu dramatique, du Faust de Marlowe à La Tempéte de Shakespeare.

\author{
M.T. jones-Davies
}

Présidente de la Société Française Shakespeare 
SOCIETE FRANCAISE SHAKESPEARE Actes de Congrès.1981

\section{THEATRE ET IDEOLOGIES : Marlowe, Shakespeare}

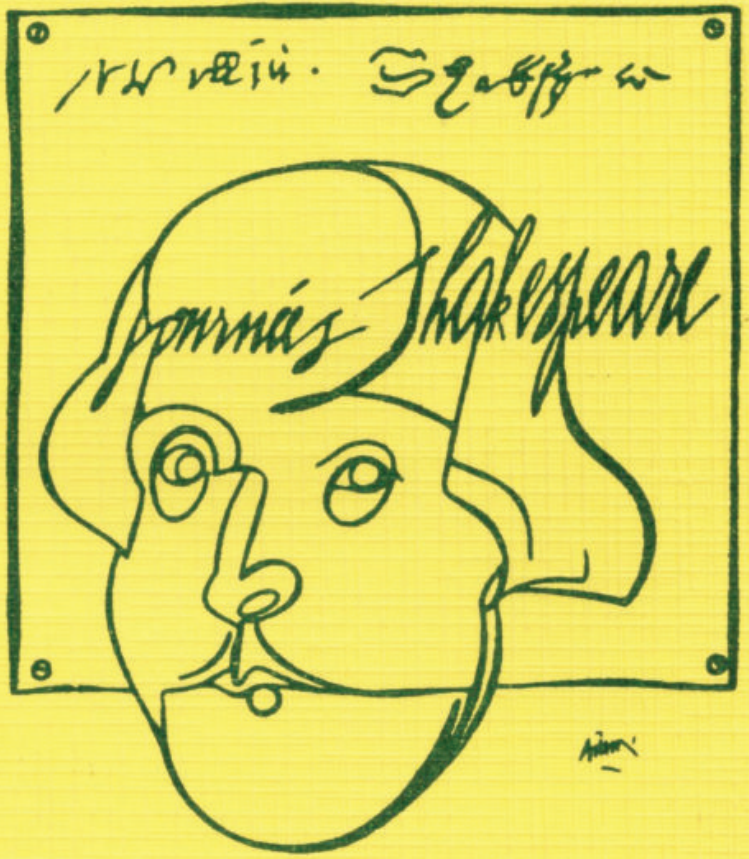

DIRECTEUR DE LA PUBLICATION M.T. Jones - Davies

JEAN TOUZOT Libraire - Editeur 38 , rue Saint-Sulpice 75278 PARIS CEDEX 061982 
Illustration de la couverture : affiche de Valerio Adami, spécialement conçue pour les Journées Shakespeare 1979 au Centre Georges Pompidou 


\section{TABLE DES MATIERES}

M.T. JONES-DAVIES Avant-propos

Théâtre et Idéologies: Marlowe, Shakespeare

S. TRUCHET

The Alchemy of Beauty: aesthetic principles in I Tamburlaine and their relationship to the hermetic ideology.

D. PRUDHOMME

L'idéologie du Juif de Malte et du Marchand de Venise.

D. GOY-BLANQUET

De Hall à Shakespeare : quelques glissements idéologiques opérés par la dramatisation dans Henry $I V$.

M.T. JONES-DAVIES Le Monıde du «si»: Idéologies Incertaines dans Comme il vous Plaira.

G. VENET

Temps et Idéologie : Marlowe, Shakespeare.

J. RICHER

Les Sept Caractères et les Quatre Tempéraments dans Jules César de Shakespeare.

F. LAROQUE

En marge de l'Idéologie : Antimasque et Grotesque dans le Dr Faustus et La Tempête.

A. LECERCLE-SWEET Conscience et Méconnaissance dans Edward The Second de Marlowe.

M.A. CONEJERO Shakespeare's Aesthe tic Scheme. (Extrait).

P.STEWART

Shylock, Shakespeare's Alien.

(Royal Shakespeare Company)

J.RUSSELL BROWN The Romantics' Shakespe are.

R. GILL

The Christian Ideology of Dr Faustus.

J. JACQUOT

Marlowe : de quelques problèmes d'interprétation. 
Rencontres entre Universitaires et Gens de Théâtre

Vendredi 4 décembre 1981
- Stuart Seide :Présentation du

Songe d'une nuit d'Eté. Mise en scène pour le Théâtre de Chaillot janvier 1982

- A propos d'Edouard (II) de

Bernard Turle

Bernard Turle : Métamorphoses de l'auteur et de l'écriture dramatiques. Intervention de Colin Harris (metteur en scène) 
Théâtre et Idéologies : Marlowe, Shakespeare.

\section{TEMPS ET IDEOLOGIE : MARLOWE ET SHAKESPEARE}

Si des considérations biographiques extérieures à l'oeuvre de Marlowe l'ont parfois emporté sur la critique interne des tragédies pour leur prêter une cohésion que les structures dramatiques démentaient, c'est peut-être qu'une dimension de cohérence manquait à l'oeuvre de Marlowe, la dimension du temps, la dimension d'un temps en accord avec les positions idéologiques prêtées aux différents personnages. Et la critique a pu penser que le temps biographique saurait ou pourrait y supléer.

La structure dramatique des tragédies de Marlowe semble en effet le lieu d'un déploiement purement spatial de l'action, une «illustration» de "moments» donnés à voir ou à entendre dans une durée neutre, informe, plutôt qu'une organisation de séquences temporelles orientées vers une fin et trouvant dans cette fin leur finalité.

Ainsi Tamerlan, au terme de son parcours conquérant, ayant goûté dans sa savoureuse plénitude le fruit de la contingence - «the sweet fruition of an earthly crown» (I; II, vii, 29) - ne découvre pas dans la mort l'obstacle transcendant toute conquete, l'obstacle temporel qui le contraindrait à modifier le sens de cette conquête, à la projeter dans une dimension autre qu'uniquement spatiale. Sur la carte du monde déployée pour la dernière fois devant lui, c'est en effet en termes d'espace que cette mort s'inscrit "And shall I die, and this unconquered ?» (II; V, iii, 150; 158). C'est à peine si le siège de Damas a pu dans cet espace infléchir un instant la linéarité d'un destin -. "Here at Damascus will I make the point / That shall begin the perpendicular" (I; IV, iv, 79-80). C'est à peine si la tentation d'abandon-«a doubful battle with my tempted soul»- a pu offrir une pause suffisante dans l'espace pour provoquer le temps d'un conflit avec Zénocrate - " you lay more siege unto my soul/ Than all my armies at Damascus'walls" (I; V, i, 155-156). Conflit fugitif pourtant dans ses effets puisque le déploiement des couleurs fatales suffit à résoudre la crise, transcription visuelle d'une impossibilité à dramatiser le conflit dans la durée, à imposer au temps une forme tragique. C'est en termes d'espace, d'espace quantitative- 
ment perçu, que s'était posé le projet de Tamerlan dès la première pièce - «I'll win the world at last» (I; III, iii, 260). C'est encore en termes d'espace, quantitativement biasphématoire, que Tamerlan perçoit sa conquête à l'acte IV de la seconde pièce - «Ready to levy power against [Jove's] throne/ For earth and all this airy region/ Cannot contain the state of Tamburlaine» (II; IV, i, 116-119). L'espace, tout entier identifié avec l'univers contingent, peut finalement entrer en rivalité avec l'univers de la transcendance cette rivalité demeure jusqu'au bout quantitative, sans prêter de qualité au temps.

C'est pourtant d'une rivalité, explicite ou tacite, entre contingence et transcendance qu'a pu naitre la tension temporelle particulière au tragique anglais. C'est de la rencontre conflictuelle entre des durées dramatiques concernées par la seule contingence et des contrain tes temporelles qu'imposait l'idéologie d'un temps perçu comme tout entier soumis à la Transcendance d'où tout hasard, tout accident, était exclu, c'est de cette rencontre qu'a pu prendre forme l'expérience temporelle du tragique anglais - expérience «existentielle» en son sens plein car n'existant qu'au sein d'un conflit tragique en mouvement et seulement pleinement dans cette période historique qui, de Marlowe à Webster, voit naitre et mourir le tragique anglais. Comme si ce tragique était à lui seul métaphore existentielle d'une irréversible métamorphose, celle de la relation de l'homme au temps. Comme si, à l'aube des «Temps Modernes», il fallait inventer un temps de la modernité, un temps dans lequel les durées contingentes cesseraient d'être le lieu indifférent d'une mutabilité dénigrée, celle de la Moralité médiévale ou de l'ontologie augustinienne, pour devenir au contraire le temps privilégié d'une action sinon déjà d'une pensée sur le mouvant. L'initiative individuelle, sous le masque de la transgression tragique, s'inventait un temps propre, le "temps humain».

Marlowe a sa part de cette invention. Non pas par un subtil déséquilibre créé au creux de la durée dramatique entre des destinées singulières et les nécessités d'une temporalité transcendante - celles que peut imposer l'idéologie "providentialiste» de l'Histoire ou de la Justice, ou celles, comminatoires, des tragédies de vengeance - subtil déséquilibre, lent déséchaffaudage des durées familières, des 
motifs temporels repris ou répétés, qui semble le privilège de l'univers shakespearien. La capacité inventive de Marlowe est autre, plutôt comme le choc titanesque d'un savoir et d'une intuition, d'un savoir idéologique sur le temps transcendant, le «temps chrétien», et d'une intuition des possibilités subversives, créatrices, de la contingence. Même s'il y a seulement mise en regard de ce savoir et de cette intuition dans l'espace de la scène plutôt que mise en conflit dans la durée dramatique, l'oeuvre de Marlowe est instauratrice d'une nouvelle relation.

Ainsi la tentation de Faust est-elle assimilable à une tentative de s'assurer une durée autonome, hors-temps en quelque sorte, hors du temps restrictif de la prédication médiévale, du temps des "Morality plays», où seule l'abstinence de toute initiative singulière pouvait protéger «Mankind» ou «Everyman» des séductions de "New Guise» ou de "New Thought», des séductions perverses de la modernité. La durée faustienne, la durée autonome du bail, si désiroires que paraissent ses vingt-quatre ans de sursis au regard de l'éternité sans limite qui en sera le prix - «no end is limited to damned souls" - cette durée se veut exploration jubilatoire - «my four and twenty years of liberty/ I'll spend in pleasure and in dalliance»--, exploration des possibles par delà les limites restrictives des savoirs codifiés, des savoirs autorisés, exploration par la magie d'un savoir qui s'étende aussi loin que peut atteindre l'esprit humain libéré de tout interdit transcendant - "stretched as far as doth the mind of man». Pourtant si cette durée exploratoire conduit Faust jusqu'aux confins de l'espace imaginaire accessible à l'esprit humain, jusqu'au Primum Mobile, jusqu'au Premier moteur initiateur du temps cosmique des "divines astrologies», elle ne le conduit pas au coeur du temps. Bien au contraire, par l'ẹxtrême mobilité que créent dans cette durée le récit des parcours imaginaires ou la labilité de Faust dans l'espace, du siège impérial au siège pontifical, des embuscades rustiques aux étreintes factices de la légendaire Hélène, toute ponctuation temporelle s'évanouit. $\mathrm{Ne}$ reste plus qu'un «interlude» aux «tableaux» multiples, vacuité exemplaire dont l'idéologie médiévale aurait fait un exemple moral, l'Oisiveté, et dont le prix pour Faust sera cette autre durée vacante qu'évoque Méphistophéles, la durée infernale dans un espace non circoncrit, un espace 
sans qualité - «Hell hath no limits nor is circumbscribed/ In one self place; but where we are is hell/ And where hell is, there must we ever be». Vertigineuse absence de repères dans l'espace et dans le temps vécue dans l'effroi et la discordance des idéologies - c'est en chrétien, en chrétien damné, que Faust dans son dernier monologue se heurte au «mur du temps», subit la violence d'une nécessaire soumission à la transcendance, c'est en chrétien rejeté et non en profane conquérant de la contingence. Mais vertigineuse absence de repères qui pourrait annoncer ce qui sera pour Webster l'audacieuse et sereine exploration de la déréliction, l'absence de toute référence possible à une transcendance, l'exploration tragique dernière, celle de $\mathrm{La}$ Duchesse d'Amalfi.

Dans l'univers de Marlowe, il semble bien que ce soit sur le temps et dans le temps qu'ait porté l'interdit. Et que l'intuition d'une force dissolvante au sein des durées contingentes n'ait pu se formuler autrement que par une dislocation des continuités narratives sans dépassement vers la création de structures temporelles originales comme ce sera le cas avec le tragique shakespearien. L'Edward II de ce point de vue est remarquable : aux antipodes des architectures historiques de Shakespeare, le récit y est réduit à l'accident, au caprice, à la décision instantanée sans postérité dans la durée, comme si une volonté subversive de casser la finalité de l'événement était à l'oeuvre, une volonté de poser, face aux interprétations rationnelles, providentielles de l'histoire, l'absence radicale de toute téléologie dans l'événement humain, le refus de toute idéologie pouvant imposer un sens à l'histoire. Dans Massacre at Paris, de la même façon, des ambitions rivales, des intrigues discontinues se juxtaposent. dans des durées parfois simplement parallèles. Une vision potentiellement subversive opposée à l'histoire comme "plan de Dieu» sur le monde aurait pu motiver le privilège soudain attribué au hasard ou à l'arbitraire des désirs ou des violences infligées. Mais chez Marlowe il semble que l'histoire s'égare dans le pamphlet anecdotique, sans jamais créer vraiment, dans la durée disloquée, la légitimité d'une mise en question idéologique, dút-elle reposer sur la revendication de la contingence, de l'événement accidentel, comme seul mode d'être dans le temps, contre tous les finalismes de la tradition historique. 
Jamais la tentation de légitimer de possibles subversions n'arrive à suggérer un point de fuite dans la durée, une perspective temporelle organisée. Comme si, des affrontements idéologiques qui tout au long du XVIe siècle avaient commencé de desserrer les liens entre cité terrestre et cité céleste, on ne retrouvait dans Marlowe que l'écho verbal, sans répercussion sur la création d'une nouvelle temporalité tragique. On hésite à conclure : soit que Marlowe ait bien tenté de capter des idéologies en mouvement, s'opposant aux utopies finalistes, aux justifications du hasard, à une «idée de nature» sur laquelle faire reposer «de droit» la légitimité d'un pouvoir; mais qu'alors il n'ait disposé que de structures dramatiques bloquées, archaiques dans leur formulation de la durée - durée amorphe qui n'autorisait que la juxtaposition, l'association brutale de données antinomiques, durée de la stychomythie médiévale, de l'opposition terme à terme, ou encore, durée médiévale elle aussi, celle de la mutabilité pure rebelle à toute interprétation dans la durée, celle des récits classiques ou des "complaintes» de Lydgate. Soit encore, autre hypothèse, que chez Marlowe comme plus tard chez Chapman, le Chapman de Revenge of Bussy d'Ambois, la volonté systématique de mettre en jeu des présupposés idéologiques ait bloqué le libre jeu dramatiques des durées et interdit l'accès à une formulation tragique du temps. Dans l'oeuvre de Chapman citée, systématique stoicienne et systématique platonicienne se conjuguent pour créer un héros théorique, Clermont, inapte à l'action tragique, pour créer une pièce à thèse, réflexion sur l'action, inapte à épouser les thèses de l'action tragique.

C'est que peut-être, dans la complexe relation qu'entretiennent à chaque époque idéologie et esthétique, c'est-àdire système d'idéation dans lequel une époque se pense et praxis imaginaire dans laquelle une époque se projette, le tragique, la praxis tragique, occupe une position singulière. Comme si justement la métaphore tragique était chargée de traduire l'expérience de l'impensable, c'est-à-dire la défaillance des idéologies, sans prévoir pour autant, sans laisser espérer comme nécessaire, un nouveau système d'intelligibilité.

A ce titre, le tragique shakespearien est exemplaire qui 
nous conduit "d'un monde clos à un univers infini» monde clos des nécessités providentielles où le temps tenait captive l'initiative individuelle ou ne la libérait que pour mieux servir le plan de Dieu sur l'histoire, c'est le cycle des usurpations géniales et des restaurations légitimes, cycle encore garanti par une mathémathique céleste, cosmique, image mobile de l'éternité. De ce monde clos, l'infléchissement progressif des durées shakespeariennes nous conduit à l'univers infini de l'improbable, de l'imprévisible «anomalie» baroque, univers d'une contingence tantôt glorifiée, tantôt banalisée mais dont "l'infinie variété» échappe à toute norme, l'univers décentré, élliptique, d'Antony and Cleopatra - dernière pièce sur l'histoire et anti-thèse des idéologies providentialistes où César restaure sans gioire la "paix universelle». Car la vraie gloire est ailleurs, la gloire baroque, dans cette trouée lumineuse vers d'hypothétiques nouveaux ciels et nouvelles terres que figure l'attente de Cléopâtre - «I have immortal longings» - et dont aucune idéologie constituée ne peut rendre compte.

Au contraire de Marlowe, c'est en effet dans des structures multiples, flexibles, que Shakespeare a noué puis dénoué les liens de la durée contingente et des temporalités transcendantes, condensé l'expérience tragique du multiple dans un temps en perpétuelle métamorphose, jusqu'à opérer la métamorphose de la relation au temps. C'est en oeuvrant subtilement dans la matière mouvante, la durée dramatique, plutôt qu'en attaquant de front le matériau monolithique d'une idéologie qu'il a réussi cette prouesse de l'esthétique paradoxale, de l'esthétique tragique : mettre en porte à faux des idéologies jusqu’à provoquer implicitement leur mise en question. Si dans la mort de Cordelia - une mort hors scène, hors centre, hors temps en quelque sorte - nous pouvons identifier la mort de l'idéologie providentielle, la fin d'une promesse sur le temps, c'est que dans les durées dramatiques qui précèdent, dans les durées dramatiques qui, de Henry $V I$ à King Lear, plient et replient sur elle-même la thématique de l'usurpation et de la légitimité, l'idéologie originelle, archaïque, d'un temps "vigilant» qui «rétribuait» chacun selon son mérite, d'un temps "rationnel», intelligible, cette idéologie au fil des durées, au contact des diverses transgressions temporelles, s'est délitée. Après l'impeccable mécanique providentielle de Richard $I I I$, déjà 
avec Richard II et Henry IV s'opère la mise en déséquilibre de deux modes d'être dans le temps, grâce aux plasticités de la durée dramatique. Dans Richard II, la légitimité, théoriquement infrangible, d'un pouvoir inefficacement géré, celui de Richard, se fragilise au contact d'une usurpation, celle de Bolingbroke à qui l'efficacité politique, la loyauté envers la communauté finalement prête une légitimité. Comme si les transactions déloyales du pouvoir légitime, les infidélités de Richard, mises au jour dans la durée dramatique qui sert à mettre en scène l'usurpation, déplaçaient le centre de culpabilité, déséquilibraient la certitude d'une restauration nécessaire. Le double jeu de la légitimité et de la rébellion, du temps et de la durée, continue dans Henry $I V$, subtilement inversé cette fois : le temps d'une restauration "providentielle» c'est-à-dire punitive plane sur la légitimité difficilement acquise d'Henry Bolingbroke, désormais Henry IV, pour qui chaque rébellion matée, chaque infraction de son fils, le Prince Hal rebelle à toute règle, est occasion de méditer sur la fragilité d'un pouvoir illégitime. L'efficacité politique de sa gestion du royaume, valorisée dans la durée dramatique par ses victoires sur la rébellion, dans la temporalité providentielle se voit mise en échec, échec à assurer dans le temps de l'histoire la durée d'une réussite, ceile d'une initiative individuelle, dans un «temps humain».

L'idéologie providentielle se survivra sous une forme résiduelle dans la "tragédie de vengeance», imposant au temps une implacable symétrie - «Blood cries for blood, and murder murder craves». Mais la netteté du projet providentiel avec Julius Caesar et Hamlet déjà suffisamment s'estompe pour que le temps de la vengeance, tout en assurant encore dans la durée dramatique la présence structurante d'une transcendance, perde toute valeur de référence à un temps historique, à un temps idéologique. Le schéma temporel de la vengeance devait permettre le passage de l'historique au symbolique, de l'idéologique au tragique. Paradoxalement, en incarcérant le temps humain de l'initiative ou de l'action dans d'autres formes moins identifiables de la nécessité - «Denmark is a prison/ - Then is the world one» - le schéma temporel de la vengeance libérait le tragique d'une systématique idéologique paralysante. Le tragique 
pouvait se faire exploratoire d'une autre relation au temps, une relation dans laquelle la "prédestination» jouerait un rôle différent.

Ce n'est pas un hasarà si la première formulation tragique de Shakespeare à s'incarner dans une structure de vengeance est Hamlet où un temps «out of joint» permet au "phénomène du tragique» de prendre le relais de la rationalisation; où la durée d'Hamlet, vécue comme vacuité contingente, creuse au coeur du temps cette «lacune» du sens dont J. Starobinski se demande si elle n'est pas l'essentiel du sens tragique de cette pièce, "de cet univers - cosmique, politique, psychologique - traversé de lacunes». Il y a un monde, un monde d'expérience tragique, entre la vacance créée dans la durée faustienne par l'idéologie, voire la contre-idéologie, et la «lacune» idéologique qui dans Hamlet autorise le tragique, ce tragique dont Clément Rosset nous assure pourtant qu'il possède une logique transcendant toute idéologie, «la logique du pire». 


\section{LA STRUCTURE ASTROLOGIQUE DU «JULES CESAR» DE SHAKESPEARE}

Les personnages dans le zodiaque, les sept caractères, les quatre tempéraments

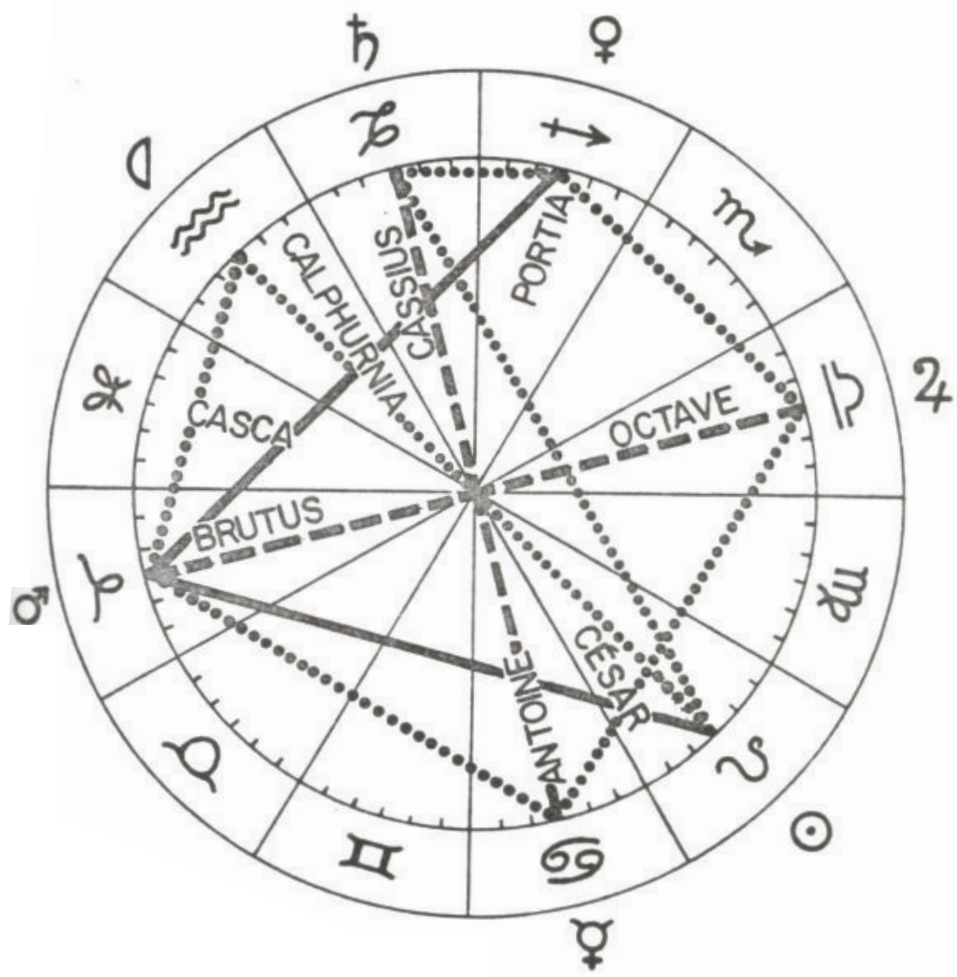

